\title{
Changing core beliefs with trial-based cognitive therapy may improve quality of life in social phobia: a randomized study
}

Vania Bitencourt Powell, ${ }^{1}$ Olivia Haun de Oliveira, ${ }^{2}$ Camila Seixas, ${ }^{1}$ Cláudia Almeida, ${ }^{1}$ Maria Conceição Grangeon, ${ }^{1}$ Milke Caldas, ${ }^{1}$ Thaís Delavechia Bonfim, ${ }^{1}$ Martha Castro, ${ }^{1}$ Amanda Galvão-de Almeida, ${ }^{1}$ Roberta de Oliveira Moraes, ${ }^{1}$ Donna Sudak, ${ }^{3}$ Irismar Reis de-Oliveira ${ }^{1}$

${ }^{1}$ Department of Neuroscience and Mental Health, Universidade Federal da Bahia (UFBA), Salvador, BA, Brazil. ${ }^{2}$ Universidade Federal de São Paulo - Escola Paulista de Medicina (UNIFESP-EPM), São Paulo, SP, Brazil. ${ }^{3}$ Department of Psychiatry, Drexel University, Philadelphia, PA, USA.

\begin{abstract}
Objective: To determine whether there are differences in quality of life (QoL) improvement after treatment with the trial-based thought record (TBTR) versus conventional cognitive therapy (CCT) in patients with social anxiety disorder (SAD).

Method: A randomized trial comparing TBTR with a set of CCT techniques, which included the standard 7-column dysfunctional thought record (DTR) and the positive data log (PDL) in patients with SAD, generalized type.

Results: Repeated measures analysis of variance (ANOVA) revealed a significant time effect in the general health, vitality, social functioning, and mental health domains of the Short Form 36. It also indicated significant treatment effects on the bodily pain, social functioning, role-emotional, and mental health domains, with higher scores in the TBTR group. One-way analysis of covariance (ANCOVA), using pretreatment values as covariates, showed that TBTR was associated with significantly better QoL post-treatment (bodily pain, social functioning and role-emotional) and at follow-up (role-emotional). A significant treatment effect on the role-emotional domain at 12-month follow-up denoted a sustained effect of TBTR relative to CCT.

Conclusion: This study provided preliminary evidence that TBTR is at least as effective as CCT in improving several domains of QoL in SAD, specifically when the standard 7-column DTR and the PDL are used.
\end{abstract}

Keywords: Cognitive therapy; randomized trial; social anxiety disorder; social phobia; quality of life; trial-based cognitive therapy; trial-based thought record

\section{Introduction}

Social anxiety disorder (SAD), also called social phobia, is a chronic and disabling condition. It is assumed to be the most common anxiety disorder, with a lifetime prevalence of $12 \%,{ }^{1}$ and a 12 -month prevalence of $7 \%$. A total of $70 \%$ of cases are categorized as severe or moderate. ${ }^{2}$ Cognitive behavioral therapy (CBT) has been shown to be effective in SAD, ${ }^{3}$ but 40 to $50 \%$ of patients show little or no improvement after treatment. ${ }^{4}$ Although CBT has recently been refined to target more specific cognitive processes believed to maintain and exacerbate symptomatology, ${ }^{5,6}$ leading to greater reductions in selfreported social anxiety symptoms than earlier protocols, newer cognitive behavioral strategies are still needed to maximize treatment efficacy. ${ }^{7}$

Correspondence: Irismar Reis de-Oliveira, Departamento de Neurociências e Saúde Mental, Largo do Terreiro de Jesus, $s / n^{\circ}$, Centro Histórico, CEP 40026-010, Salvador, BA, Brazil.

E-mail: irismar.oliveira@uol.com.br

Submitted Apr 06 2012, accepted Oct 202012.
Quality of life (QoL) is highly compromised in SAD. ${ }^{8-10}$ In a sample of predominantly untreated patients with SAD symptoms recruited over two decades, QoL was significantly reduced in both pure and comorbid SAD, especially in scales measuring general health, rolelimitations due to emotional health, social functioning, general mental health, and vitality. Together, at least 50\% of all patients suffering from moderate and severe SAD had a markedly reduced QoL, compared to $4.6 \%$ in the control group. ${ }^{9}$ Studies suggest that even patients with SAD who do not have significant comorbid depression or anxiety are substantially impaired in mental health and social functioning. ${ }^{10}$

Studies assessing QoL in SAD after CBT are scarce. However, the impact of SAD on self-perceived QoL immediately after treatment ${ }^{8}$ and at 6 -month follow-up have been investigated. ${ }^{11}$ Studies suggested that patients with SAD had very low self-rated QoL scores, which varied with symptomatic distress and impairment. This research has also found that socially anxious patients showed significant improvement in self-perceived QoL immediately after cognitive-behavioral group therapy (CBGT). ${ }^{8}$ Such 
gains were maintained from post-treatment to 6-month follow-up. ${ }^{11}$ A more recent study of SAD patients who received 12 to 20 2-hour CBGT sessions concluded that, although symptoms of SAD and some aspects of QoL improved for up to 12 months after CBGT, the social functioning domain did not change, suggesting the need for better treatments for this condition. ${ }^{12}$

The trial-based thought record (TBTR), ${ }^{13}$ a novel approach used in trial-based cognitive therapy (TBCT), ${ }^{14}$ was developed to address negative core beliefs (CBs) in CBT. In TBTR, the therapist engages the patient in a simulation of a legal trial, stimulating the activation of negative CBs and associated negative emotions, with the aim of reducing their effect with disconfirming evidence. The repeated use of TBTR may eventually deactivate negative dysfunctional CBs, as more credible evidence in support of functional CBs is produced. ${ }^{15}$

In the present study, we hypothesized that TBTR would reduce symptoms of social anxiety by restructuring CBs. The objective was to assess the short- (4-month) and long-term (12-month) efficacy of TBTR in SAD. Findings regarding QoL are reported in the present study, while a detailed description of results involving other clinical outcomes has been previously published. ${ }^{16}$

\section{Methods}

The method used in this study is described in greater detail elsewhere, ${ }^{16}$ but a brief description of the main methodological aspects is provided below.

Advertisements in local newspapers and interviews about social anxiety given by the first author to local radio and television stations were used to recruit the patients. Only people who met DSM-IV criteria for generalized SAD were included in the study. All patients were assessed at an anxiety disorders clinic in a university teaching hospital.

Forty-seven patients who completed initial assessments were randomized to treatment, with 25 of these allocated to TBTR and 22 to conventional cognitive therapy (CCT) (Figure 1). However, differently from the clinical outcomes described elsewhere ${ }^{14}$ the final sample for QoL analyses in the present study included only the 16 patients in the TBTR group and 14 in the CCT group who had attended all 12 treatment sessions.

The study was a two-arm randomized trial comparing TBTR with a set of CCT techniques, which included the standard dysfunctional thought record (DTR) and the positive data log (PDL). ${ }^{17}$ The researcher conducting randomization was blind to the treatment protocol. After signing the informed consent form, participants were assessed with the Mini International Neuropsychiatric Interview (MINI). ${ }^{18}$ Participants of any gender or ethnic group, aged 18 to 70 , able to read and write, who fulfilled DSM-IV criteria for SAD, generalized type were included in the study. Exclusion criteria comprised major comorbid Axis I psychiatric disorders (e.g., major depression, schizophrenia, bipolar disorder), alcohol or substance use/abuse in the past 6 months, suicide risk, or presently being in psychotherapy.
Treatment consisted of 10 weekly 1 -hour individual sessions of either TBTR (experimental group) or CCT, and two 1-hour biweekly sessions in the last 4 weeks. Sessions 1 to 5 in both treatment conditions involved psychoeducation about the cognitive model and cognitive distortions. From session 6 onward, both treatments focused on restructuring CBs with either the TBTR or CCT, specifically the standard 7-column DTR and the PDL. TBTR techniques differed from the CCT techniques in that (in addition to simulating a legal trial) patients were actively stimulated to disqualify the evidence that did not support the negative CBs (e.g., "I am awkward"). Afterwards, by means of a sentence-reversal approach, the therapist engaged the patients in a second round exploring the evidence that supported the positive CBs (e. g., "I am normal"). TBTR also differed from the CCT techniques in that in TBTR the new positive CBs were uncovered by means of the upward arrow technique. From sessions 6 through 12, both approaches (TBTR and CCT) were focused on uncovering and bolstering the new positive CBs. Although the DTR was not designed by Beck et al. ${ }^{19}$ or modified by Greenberger and Padesky ${ }^{20}$ to change CBs, it is one of the most commonly used techniques for this purpose. Furthermore, the PDL was specifically designed to change $\mathrm{CBs},{ }^{17}$ which is why it was included in the contrast group.

All the therapists were psychologists and certified cognitive therapists previously trained in both approaches. Therapists had attended a 2-year cognitive therapy specialization course organized by two of the authors (IRO and VBP). They were invited to participate in the study because they were among those who had the best performance during training. The course included $384 \mathrm{~h}$ of theory, $60 \mathrm{~h}$ of clinical experience with patients and $86 \mathrm{~h}$ of supervision. Also, knowledge and competence were assessed through a total of 23 monthly written exams throughout the course. In addition to having completed the specialization course, all therapists had at least 1 year of experience in private practice as certified cognitive therapists at the time the clinical trial began. All therapists received training together, so that therapists in both arms (i.e., TBCT and CCT) had equivalent experience and expertise in both approaches.

Assessment of QoL was performed with the Brazilian version $^{21}$ of the Short Form 36 (SF-36), ${ }^{22}$ a 36 -item selfreport questionnaire addressing the following domains: physical functioning, role-physical, bodily pain, general health perception, vitality, social functioning, roleemotional and mental health. The questionnaire was administered at pre-treatment, post-treatment (4 months), and 12-month follow-up.

All patients who provided at least two SF-36 assessments (baseline and post-treatment) were included in the analyses (completers). However, a last observed carried forward (LOCF) intention-to-treat approach was employed for the 12-month follow-up analyses, so that analyses included completers at post-treatment and intention-to-treat (ITT) at follow-up.

A mixed (repeated measures) analysis of variance (ANOVA) was implemented to evaluate the efficacy of the 


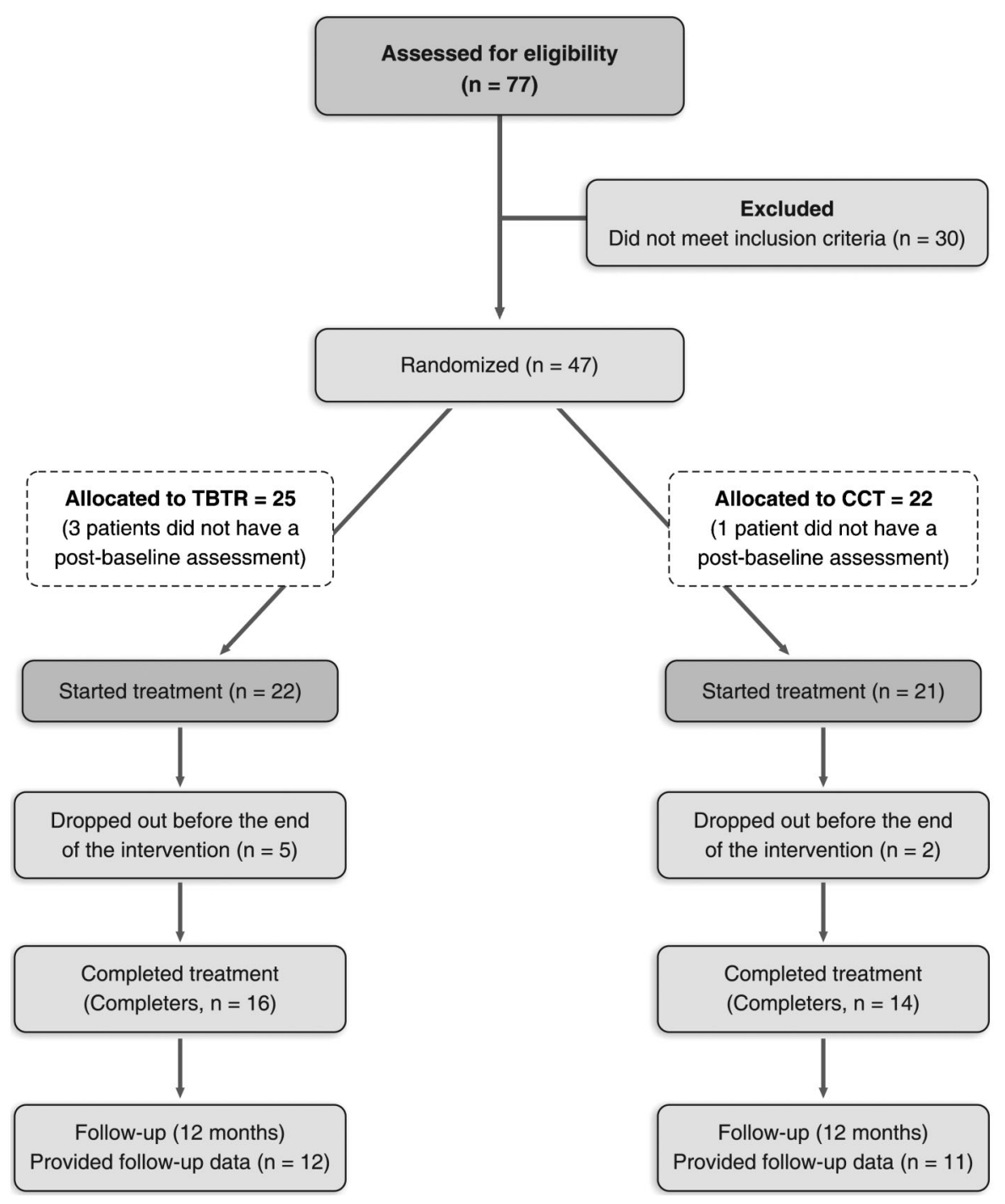

Figure 1 Flow diagram of participants through stages in the randomized trial. CCT = conventional cognitive therapy; TBTR= trial-based thought record.

interventions, with the number of assessments (pretreatment, post-treatment and follow-up) being computed as a within-subject factor (time) and treatment modality (TBCT and CCT) as a between-subjects factor. We also conducted a one-way analysis of covariance (ANCOVA) using baseline scores as covariates. Level of significance was set at .05. Analyses were conducted with the SPSS 17.0 software.

Within-subjects Cohen's $d$ effect sizes were also calculated. ${ }^{23}$ Effect sizes (ESs) of 0-0.32 were considered small, scores of 0.33-0.55 were considered moderate, while those between 0.56 and 1.2 were interpreted as large. ESs greater than 1.2 were assumed to be very large.

\section{Results}

A mixed (repeated measures) ANOVA revealed a significant time effect in the general health, vitality, social functioning and mental health domains of QoL, and the results of this analysis are displayed in Table 1. This table also displays significant treatment effects in the bodily pain, social functioning, role-emotional, and mental health domains, with higher scores in the TBTR group. A oneway ANCOVA, taking pre-treatment values as covariates, showed that TBTR led to significantly better posttreatment (bodily pain, social functioning and role-emotional) and follow-up (role-emotional) results than the CCT techniques. There was also a significant treatment effect on the role-emotional domain at follow-up, showing a sustained effect of TBTR relative to CCT.

Table 2 shows Cohen's $d$ ESs for both TBTR and CCT.

\section{Discussion}

The present study shows that both CCT and TBTR, a novel cognitive behavioral approach, ${ }^{24}$ improved several domains of QoL, namely general health, vitality, social 
Table 1 Quality of life measures according to the SF-36 (TBTR, $n=16$; CCT, $n=14$ ) at baseline, post-treatment and 12-month follow-up (mean and SD)

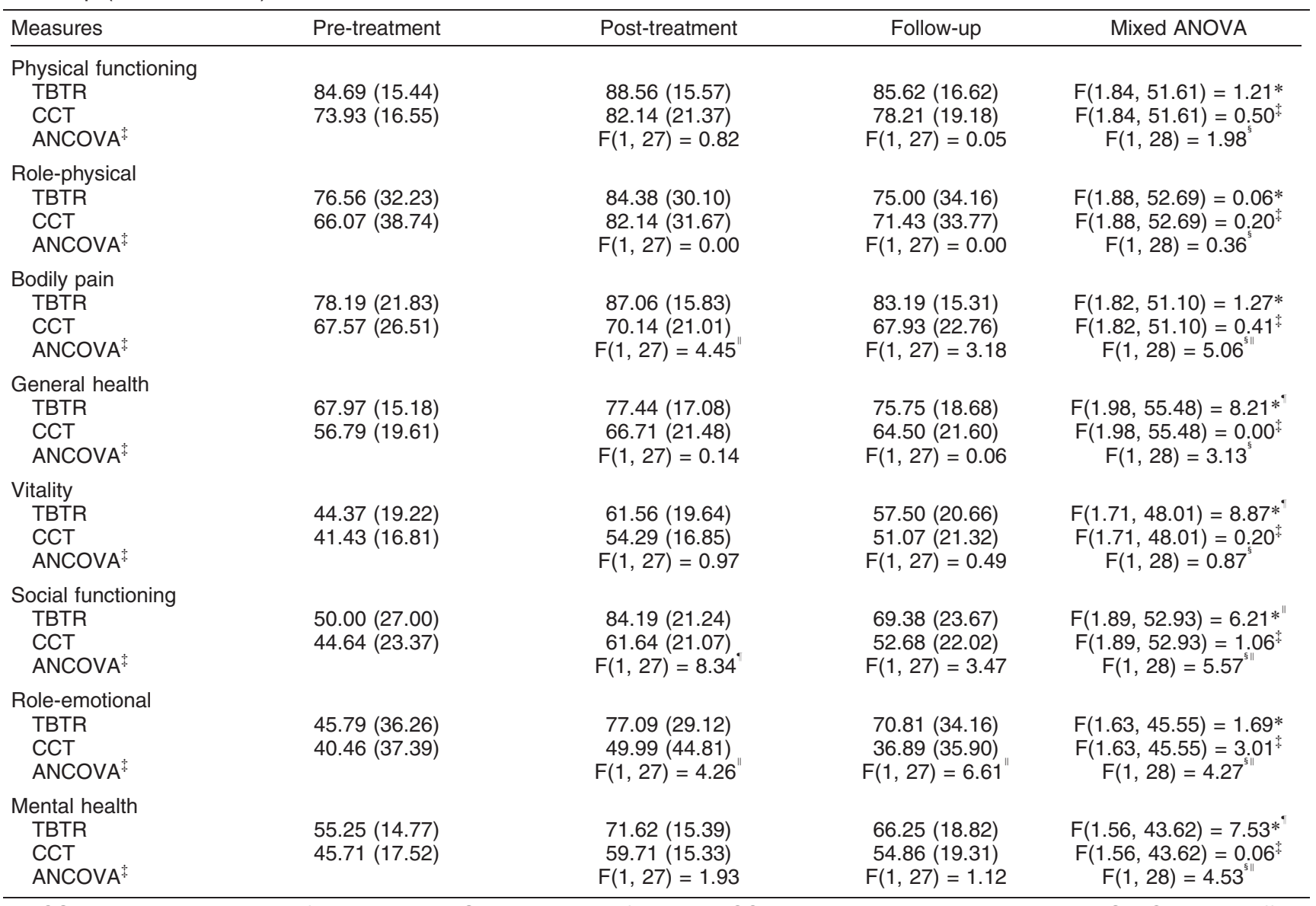

ANCOVA = one-way analysis of covariance; ANOVA = analysis of variance; CCT = conventional cognitive therapy; ES = Cohen's $d$ effect size; SD = standard deviation; SF-36 = Short Form 36; TBTR = trial-based thought record.

* Overall time effect.

$\dagger$ Interaction (time $\mathrm{x}$ treatment) effect.

ANCOVA with pretreatment assessments as covariates.

$\S$ Treatment (group) effect.

$\| p \leqslant 0.05 ; " p \leqslant 0.01$.

functioning, and mental health. As far as we know, this is the first study comparing QoL measures following a new CBT approach with standard CBT techniques in SAD. Moreover, we are aware of no studies comparing the long-term outcomes of different CBT approaches.
An earlier study ${ }^{12}$ of SAD patients found modest improvement in aspects of QoL following treatment, which were maintained at the 12-month follow-up. The Cohen's $d$ ESs seen in the CCT group in the present study (Table 2) were similar or slightly larger than those

Table 2 Cohen's $d$ effect sizes found in this study and in Watanabe et al. ${ }^{21}$

\begin{tabular}{lccc}
\hline SF-36 domains & CCT, post-/F-U & TBCT, post-/F-U & Watanabe et al., post-/F-U \\
\hline Physical functioning & $0.43 / 0.24$ & $0.25 / 0.06$ & $0.10 / 0.15$ \\
Role-physical & $0.45 / 0.15$ & $0.25 / 0.05$ & $0.06 / 0.39$ \\
Bodily pain & $0.11 / 0.01$ & $0.47^{*} / 0.27$ & $0.24 / 0.12$ \\
General health & $0.48 / 0.37$ & $0.59 / 0.46$ & $0.28 / 0.44$ \\
Vitality & $0.76 / 0.50$ & $0.88 / 0.66$ & $0.51 / 0.29$ \\
Social functioning & $0.76 / 0.35$ & $1.41^{*} / 0.76$ & $0.30 / 0.28$ \\
Role-emotional & $0.23 /-0.10$ & $0.95 * / 0.71^{*}$ & $0.07 / 0.08$ \\
Mental health & $0.85 / 0.50$ & $1.09 / 0.65$ & $0.39 / 0.32$
\end{tabular}

$\mathrm{CCT}=$ conventional cognitive therapy; TBTR = trial-based thought record; post- = post-treatment; F-U = follow-up.

$* p \leqslant 0.05 ; p \leqslant 0.01$. 
found in the aforementioned study. ${ }^{12}$ However, TBTR had large to very large ESs at post-treatment in the mental health $(E S=1.09)$ and social functioning $(E S=1.41)$ domains, as well as large ESs in the social functioning domains $(E S=0.76)$ at follow-up, and these results were not accomplished neither in the CCT contrast group of our study nor in that study. ${ }^{12}$ A possible explanation for this finding is that TBTR had a more significant effect on fear of negative evaluation (FNE) scores than CCT techniques, as shown in a previous publication of this study. ${ }^{16}$ The one-way ANCOVA showed a significant treatment effect $(F[1,33]=5.47 ; p=0.03)$ at midtreatment and at post-treatment, with participants in the TBTR group scoring lower on FNE assessments than participants in the CCT group.

Clearly, results from this study should be interpreted in light of its limitations. Firstly, the sample size was small. Secondly, patients were recruited by means of advertisements and interviews on radio and television, which may mean this sample is not representative of the treatmentseeking SAD population, even though all participants fulfilled DSM-IV criteria for generalized SAD. Thirdly, all patients received the same intervention in the initial phase of the study (i.e., first 5 sessions), so the comparison is based on a short duration of treatment (weeks 6 to 12). Nevertheless, even with this short duration of treatment, significant differences emerged on several SF-36 domains. A final limitation was that comparison was performed with a contrast group rather than a control group. The latter would have added value to this study.

In summary, this pattern of findings allows us to conclude that TBTR is significantly more effective than CCT, specifically the standard 7-column DTR and the $\mathrm{PDL}$, in improving several domains of QoL in the short(namely bodily pain, social functioning and role-emotional) and long-term (namely role-emotional). These results suggest that it would be valuable to investigate the efficacy of TBTR, as well as the broader approach, TBCT, ${ }^{24}$ in a larger sample of patients with SAD, as well as in patients with other psychiatric disorders characterized by dysfunctional CBs. ${ }^{25}$

\section{Disclosure}

The authors report no conflicts of interest.

\section{References}

1 Kessler RC, Berglund P, Demler O, Jin R, Merikangas KR, Walters EE. Lifetime prevalence and age-of-onset distributions of DSM-IV disorders in the National Comorbidity Survey Replication. Arch Gen Psychiatry. 2005;62:593-602.

2 Kessler RC, Chiu WT, Demler O, Merikangas KR, Walters EE. Prevalence, severity, and comorbidity of 12-month DSM-IV disorders in the National Comorbidity Survey Replication. Arch Gen Psychiatry. 2005;62:617-27.

3 Acarturk C, Cuijpers P, van Straten A, de Graaf R. Psychological treatment of social anxiety disorder: a meta-analysis. Psychol Med. 2009;39:241-54.

4 Heimberg RG, Liebowitz MR, Hope DA, Schneier FR, Holt CS, Welkowitz LA, et al. Cognitive behavioral group therapy vs phenelzine therapy for social phobia: 12-week outcome. Arch Gen Psychiatry. 1998;55:1133-41.

5 Clark DM, Ehlers A, McManus F, Hackmann A, Fennell M, Campbell $\mathrm{H}$, et al. Cognitive therapy versus fluoxetine in generalized social phobia: a randomized placebo-controlled trial. J Consult Clin Psychol. 2003;71:1058-67.

6 Hofmann SG, Scepkowski LA. Social self-reappraisal therapy for social phobia: preliminary findings. J Cogn Psychother. 2006;20:4557.

7 McManus F, Clark DM, Grey N, Wild J, Hirsch C, Fennell M, et al. A demonstration of the efficacy of two of the components of cognitive therapy for social phobia. J Anxiety Disord. 2009;23:496-503.

8 Safren SA, Heimberg RG, Brown EJ, Holle C. Quality of life in social phobia. Depress Anxiety. 1996-1997;4:126-33.

9 Wittchen HU, Fuetsch M, Sonntag H, Muller N, Liebowitz M. Disability and quality of life in pure and comorbid social phobia. Findings from a controlled study. Eur Psychiatry. 2000;15:46-58.

10 Simon NM, Otto MW, Korbly NB, Peters PM, Nicolaou DC, Pollack $\mathrm{MH}$. Quality of life in social anxiety disorder compared with panic disorder and the general population. Psychiatr Serv. 2002;53:714-8.

11 Eng W, Coles ME, Heimberg RG, Safren SA. Domains of life satisfaction in social anxiety disorder: relation to symptoms and response to cognitive-behavioral therapy. J Anxiety Disord. 2005;19:143-56.

12 Watanabe N, Furukawa TA, Chen J, Kinoshita Y, Nakano Y, Ogawa $\mathrm{S}$, et al. Change in quality of life and their predictors in the long-term follow-up after group cognitive behavioral therapy for social anxiety disorder: a prospective cohort study. BMC Psychiatry. 2010;10:81.

13 de-Oliveira IR. Trial-Based Thought Record (TBTR): preliminary data on a strategy to deal with core beliefs by combining sentence reversion and the use of analogy with a judicial process. Rev Bras Psiquiatr. 2008;30:12-8.

14 de-Oliveira IR. Common Language for Psychotherapy (CLP) procedures [Internet]. Trial-based thought record. 2011 [cited 2013 Jul 2]. http://www.commonlanguagepsychotherapy.org/fileadmin/ user_upload/Accepted_procedures/trial-based.pdf

15 de-Oliveira IR. Use of the trial-based thought record to change negative core beliefs. In: Standard and innovative strategies in cognitive behavior therapy. de-Oliveira IR, editor. Rijeka: InTech; 2012. p. 35-60.

16 de-Oliveira IR, Powell VB, Wenzel A, Caldas M, Seixas C, Almeida $\mathrm{C}$, et al. Efficacy of the trial-based thought record, a new cognitive therapy strategy designed to change core beliefs, in social phobia. $J$ Clin Pharm Ther. 2012;37:328-34.

17 Tompkins MA, Persons JB, Davidson J. Cognitive-behavior therapy for depression: schema change methods [DVD]. Washington: American Psychological Association; 2006. [cited 2010 Jun 24]. http://www.apa.org/pubs/videos/4310776.aspx

18 Sheehan DV, Lecrubier Y, Sheehan KH, Amorim P, Janavs J, Weiller E, et al. The Mini-International Neuropsychiatric Interview (M.I.N.I.): the development and validation of a structured diagnostic psychiatric interview for DSM-IV and ICD-10. J Clin Psychiatry. 1998;59:22-33;quiz 34-57.

19 Beck AT, Rush AJ, Shaw BF, Emery G. Cognitive therapy of depression. New York: Guilford Press; 1979.

20 Greenberger DG, Padesky CA. Mind over mood. New York: Guilford Press; 1995.

21 Ciconelli RM, Ferraz MB, Santos W, Meinão I, Quaresma MR. [Brazilian-Portuguese version of the SF-36. A reliable and valid quality of life outcome measure]. Rev Bras Reumatol. 1999;39:14350.

22 Ware JE Jr., Sherbourne CD. The MOS 36-item short-form health survey (SF-36). I. Conceptual framework and item selection. Med Care. 1992;30:473-83.

23 Borenstein M, Hedges LV, Higgins JPT, Rothstein HR. Introduction to meta-analysis. Chichester: John Wiley \& Sons; 2009.

24 de-Oliveira IR. Kafka's trial dilemma: proposal of a practical solution to Joseph K.'s unknown accusation. Med Hypotheses. 2011;77:5-6.

25 de-Oliveira IR, Hemmany C, Powell VB, Bonfim TD, Duran EP, Novais $\mathrm{N}$, et al. Trial-based psychotherapy and the efficacy of trialbased thought record in changing unhelpful core beliefs and reducing self-criticism. CNS Spectr. 2012;17:16-23. 